\title{
Development of Entering and Reporting Registration System Platform of the CAU Library in COVID-19 Epidemic Period
}

\author{
Hao YU,1 \\ ${ }^{a}$ China Agricultural University
}

\begin{abstract}
This article elaborates briefly development of entering and reporting registration system platform according to the requirements of the CAU Library to prevent and control the epidemic COVID-19. The development of the system are extremely significant in supporting the teaching progress of the university and protecting the users on the campus. It has clarified the system development principles and implement methods used in developing the system for facilitating the prevention and control of the epidemic COVID-19 on the campus including good compatibility, comprehensive user management permissions, perfect business processes, multi-mode login, and custom entering record data output. Based on the $\mathrm{B} / \mathrm{S}$ model, various open source technologies such as LAMP architecture, Bootstrap and LayUI framework, AJAX technology, JSON data exchange format, combined with QR code technology are mainly adopted in developing the system after literature review. The major system functions are realized by five functional modules: user login, application management, QR code management, entering library record management and system management. Each module carries out strict operation control according to different use-permission of users. Compared with the traditional manual record management mode, the research result shows that compared with the traditional manual record management mode, the platform management mode of the library entering and reporting registration system has the advantages of high efficiency, convenience, accuracy and reliability which achieved $100 \%$ of the library entrance registration. Because of its strong practicability and high expansibility, it has been widely adopted by other colleges of the university and achieved very good expected results.
\end{abstract}

Keywords. Reporting and registration system; LAMP; WeChat authorized login; Responsive webpage; Quick respond code technology; LayUI

\section{Introduction}

The COVID-19 has been spreading since the end of 2019. There were 10,719,946 confirmed cases, including 517,337 deaths, reported by World Health Organization (WHO) till the end of June, 2020 [1]. Currently, the entire world is struggling against the virulent pandemic COVID-19. Everyone is witnessing the unbounded global spread of the disease. The pandemic has brought a revolution not only in the online teaching of higher education but also in the effective ways academic libraries can deliver their

${ }^{1}$ Corresponding Author. Hao YU, Librarian, China. E-mail: tsg006@cau.edu.cn. This research was financially supported by Collection and Collation of Agricultural Professors and Scholars' Information Resources (Grant NO. 29026004). 
services virtually [2]. Libraries need to leverage their position as a primary source of trustworthy information by providing quick and easy access to those looking for credible information about what to do in an emergency [3]. In order to implement the China national policy guidelines for the prevention and control of the epidemic, China Agricultural University (CAU) urgently issued relevant policies to strictly control the returning of its staffs and students to the university and implemented closed management at the university.

As one of the important service departments of scientific research and education activities in universities, the library is also facing huge challenges during this epidemic prevention and control work. It is necessary to provide resource services for students of various departments in a timely manner, and also to resolutely undertake epidemic prevention and control to avoid cross-infection caused by personnel gathering and book borrowing. To this end, the China Agricultural University Library has carried out a comprehensive and meticulous deployment. One of the most important tasks is to develop a library entering and reporting registration system to provide basic guarantee for the daily management and operation and maintenance of the library. It has realized various functions such as personnel identity verification and entering registration approval as the system combines the computer and mobile terminals. Many technologies have been chosen for the development of this system, such as: LAMP, Responsive webpage, Quick respond code technology and LayUI etc. The advantages of the system clearly shows that the data of entering and leaving the library can be traced, checked and controlled, and the system is convenient and fast to use and manage.

This automatic management alternative has been adopted by many other colleges of the university which has effectively replaced the inefficient manual management model. This article comprehensively summarizes the design, development and practical application of the system.

\section{Literature review}

\subsection{Software architecture}

Browser/Server $(\mathrm{B} / \mathrm{S})$ mode and Client/Server $(\mathrm{C} / \mathrm{S})$ mode are two commonly used software development modes. In order to build an efficient, sample and convenient system of face recognition based on the video streaming, a mixed mode of software development is proposed in this research. Although some experts think mixed of $\mathrm{B} / \mathrm{S}$ and $\mathrm{C} / \mathrm{S}$ modes makes a system more flexible and convenient for user to use [4]. However, C/S mode is more suitable for LAN, special software needs to be installed in the client, so the cost of maintenance and upgrade is high. B/S mode is a kind of network structure mode, which can be realized by only one browser without installing the client. The installation and operation of the system are all operated on the server. $\mathrm{B} / \mathrm{S}$ mode has the advantages that $\mathrm{C} / \mathrm{S}$ mode can't compare with [5].

LAMP architecture is a combination of Linux, Apache, PHP and MySQL which is used under B/S mode. It is a popular web development architecture widely used in the world at present. Its web components include Linux operating system, Apache network server, MySQL database and PHP server scripting language. All components are opensource free software. Compared with Java EE and asp.net, LAMP architecture has 
higher performance, lower cost, faster development speed and more flexible execution [6].

\subsection{Bootstrap and LayUI front-end framework}

Response web design was proposed by Ethan Marcotte, a famous web designer, in 2010. Its design concept is that the design and development of the page should respond and adjust according to the user behavior and the equipment environment (system platform, screen size, screen orientation, etc.). In short, it can automatically adapt and recognize the screen size, so as to adjust the appropriate web page, which can be normally displayed on any device or on each terminal [7]. The so-called front-end framework refers to gathering of a series of productized HTML/CSS/JavaScript components. Using the front-end development framework, developers can use the least effort to create responsive websites that meet user requirements, making the whole development process simple and consistent. The Bootstrap framework has a complete set of basic modules, predefined style sheets, etc. Among them, the global grid system is responsible for the adaptive matching of display terminals of different sizes and resolutions, to ensure that the display content is presented to users in a clean and smooth manner, and to bring good experience to users [8].

Bootstrap has beautiful style and well packaged plug-ins, which is more suitable for page design, but in terms of data interaction with back-end servers, compared with the domestic front-end LayUI framework, it appears to be somewhat bloated and slow. The LayUI framework is a front-end UI framework written with its own module specification. It follows the writing and organization form of native HTML/CSS/JS. Driven by the document object model, a large number of program interfaces are built in to realize the front-end and back-end data interaction. It really achieves more functions with less code. Using it to develop the front-end data interaction can greatly shorten the development cycle, improve development efficiency [9].

\subsection{AJAX technology}

Asynchronous JavaScript and XML (asynchronous JavaScript and XML for short, AJAX) is a web development technology that creates interactive web applications. If the traditional web page needs to update the content, it must overload the whole web page, which is easy to increase the server load. When the data volume is large, the user friendliness will be greatly reduced. AJAZ technology's application in library system, implements asynchronous data transmission in the case of no page refreshing, enhances interactive of network teaching resources library system, and improves the efficiency of the network teaching resources server [10]. By using AJAX to exchange a small amount of data with the server in the background, the web page can be updated asynchronously, that is, the whole web page will not be reloaded, and only the local information of the web page needs to be updated as required, so as to achieve faster and friendly response to user operations [11].

\subsection{JSON data format}

JavaScript object notation (JSON for short) is a lightweight data exchange format with good readability and fast parsing features. JSON file is a common text file, which can be edited by using common text editor. The back-end program extracts and sends various data of different structures from the database. As long as it is converted to 
JSON format, it can be directly used to write some parts of the web page. At the same time, the analytic speed of JSON in browser is very fast, which can effectively improve the system efficiency [12].

\subsection{QR code and its RSA anti-counterfeiting technology}

QR code is a kind of black-and-white figure that storing data symbol information in horizontal and vertical directions according to certain rules. It uses a certain geometry figure on the basis of one-dimensional code. QR code has the advantages of representing a variety of text information such as Chinese character image, large storage capacity and strong reliability [13]. Theoretical perspective studies in [14] outlined that QR code is one of technologies that could be applied in library management system. It has been suggested that the use of QR code for library offer some advantages involving such as fast process, able to store big data, uses standard technology (only camera to percept the code), which can be used by user without specific skills. So it would be able to enhance service quality such as to shorten borrowing or returning transaction as well as increasing security of the books from theft in low cost.

Because QR code itself does not have anti-counterfeiting ability, in order to ensure its security, QR code and RSA anti-counterfeiting technology are better combined to make it having a high anti-counterfeiting ability. RSA algorithm was proposed by Ron Rivest, ADI Shamir and Leonard Adleman in 1977, which is called RSA algorithm for short. It is a relatively mature and perfect public key cryptosystem so far, and a typical representative of asymmetric cryptosystem. It is widely used in many aspects in network development and information security [15]. QR code of the system is completely encrypted by RSA, which makes the operation convenient, fast and efficient, while maintaining high security.

\subsection{LayUI excel component}

The downloading of files can be simply divided into two ways: fixed link downloading and dynamic program downloading. Because of the single target resources, the fixed link downloading cannot meet the system requirements, while the dynamic program downloading can be flexibly downloaded and output according to different needs, which is widely used at this stage. The traditional dynamic program download includes back-end download and front-end download according to the different location of Excel file generation. Back-end download is a more extensive way of data output and download. Its principle is that the back-end program uses the corresponding operational programs to generate Excel files in the server according to the condition settings, and then returns them to the browser in the way of data flow for export and download. The principle of this method is simple and clear, but the actual operation is relatively cumbersome and consumes server resources, which will have a harmful effect on server performance.

The realization of front-end download is based on LayUI Excel, the export component base of LayUI framework. It is a relatively simple, flexible and powerful open source component in the existing front-end operation Excel files. In the process of data export and download, less server resources are used. The back-end is only responsible for returning JSON format exchange data. The front-end LayUI excel component extensions is responsible for data format conversion, style and Excel file 
generation and download. After testing, it takes about 7 seconds [16] to output 100,000 items with 9 columns of Excel data, which is the best download and output solution that meets the needs of the system.

\section{Methods}

\subsection{Demand analysis}

With continuous mitigation of the domestic epidemic in China, especially in Wuhan, Hubei province, was lifted the lockdown, thousands upon thousands of people return to Beijing. It is necessary to carry out preliminary examination and approval of the whereabouts of the users whom entering the library, and to record detailed and qualified physical conditions of them in order to strengthen the library entering management. Most of the traditional management methods are manual records by filling out paper application forms, which was time consuming and inefficiency. All of them involve a lot of data management, search and statistical analysis. Each task requires a lot of manpower, material resources and time cost to complete, and the completion effect has yet to be tested. Such a management method not only has low efficiency, but also easy to have human error sometimes, which cannot meet the actual needs of personnel information management during the current epidemic period of time. So, an effective and quick tracing system is particularly needed during the epidemic period at the university. The system is required to be flexible and friendly to manage and update all the basic information of users entering the library, such as whereabouts registration and verification of the 14 days before the first entering of the library, and records of physical temperature and health status when entering.

Through detailed demand analysis and in-depth research for solving the above problems, China Agricultural University library has specified the functional requirements for the development of the library entering and reporting registration system platform, which mainly include a system interface with strong compatibility and good user experience; strict and complete user rights management; detailed application form and perfect business process; support multiple ways to log in; custom batch export data of library records and other functions.

\subsubsection{A system interface with strong compatibility and good user experience}

Due to uncertainties in the personal schedule and use of library equipment during the epidemic, sometimes only a small number of people use computers and most of users will use a variety of mobile devices such as Pad or mobile phones. Therefore, compatibility issues should be fully considered to provide users with a friendly system interface compatible with different browsers and screen resolutions at the beginning of system development.

\subsubsection{Strict and complete user rights management}

The users of the system mainly consist of managers, teachers, researchers and students, as well as other staffs. Strict authority management must be adopted to set different operation authorities for different types of users considering the security needs of the system. The administrator has the maximum authority and can set different usage rights 
for different users, such as: whether may apply for library entering, or have approval authority, effective use period of quick respond code (QR code), blacklist setting, etc.

\subsubsection{Support multiple ways to log in}

Users need to $\log$ in to the system using mobile phone and present the QR code when they pass the application review and prepare to enter the library. The traditional login method of accessing to the library website is tedious and error-prone as the user name and password were required to fill in. Based on the library's WeChat public account, combined with WeChat's "sweep" function for WeChat authorization login. It is easy to operate, accurate and efficient, and at the same time it conforms to the characteristics of public behavior. Thus, it is the best way to log into the system. WeChat authorized login is a WeChat OAuth 2.0 authorized login system built on WeChat OAuth 2.0 protocol standard. It can be used to quickly and securely login to third-party applications or systems through WeChat [17]. This method can be used as an effective supplement to the system login.

\subsubsection{Custom batch export data of library records}

Users system administrator needs to download and export the record data of users entering the library within a certain period of time and submit the records data according to the relevant requirements of the superior management department. Therefore, the system must have the function of batch export of data, which can be filtered and retrieved by user-defined multiple retrieval conditions, such as date range, user type etc., and batch export data set records to Excel file according to the retrieval results.

\subsection{System design}

In order to collect data and monitor the various function of the system in real time, the scheme of data visualization interface for library entering and reporting registration system is proposed based on the combination of front and rear end technologies of Internet. The system has realized various system functions by using the structural characteristics of $\mathrm{B} / \mathrm{S}$ mode with the LAMP architecture design pattern. Bootstrap and LayUI front-end framework are used to realize responsive web page design. AJAX technology is adopted for data exchange at front and back ground of the system. JSON data format is adopted for heterogeneous data exchange between front and back end of the system. QR code and its RSA anti-counterfeiting technology are implemented. Download and export technology of front-end Excel data file based on LayUI excel component are also utilized.

\subsubsection{Detailed application form and perfect business process.}

The different types of users entering the library need to be managed differently during the epidemic period. Especially for those who return to Beijing from areas where the epidemic is serious. It is necessary to grasp the information of returning to Beijing in a timely manner, accurately grasp the isolation and physical condition information before one entering the library. "Library Entering Application Form" is required to be filled in details and submitted through the system. The application needs to be reviewed by the superior, QR code needs to be scanned after passing the review before entering the 
library, physical temperature will be measured and recorded (all personal temperature records need to be kept on file for reference), and finally personal photo will be checked before entering the library (see Figure 1).

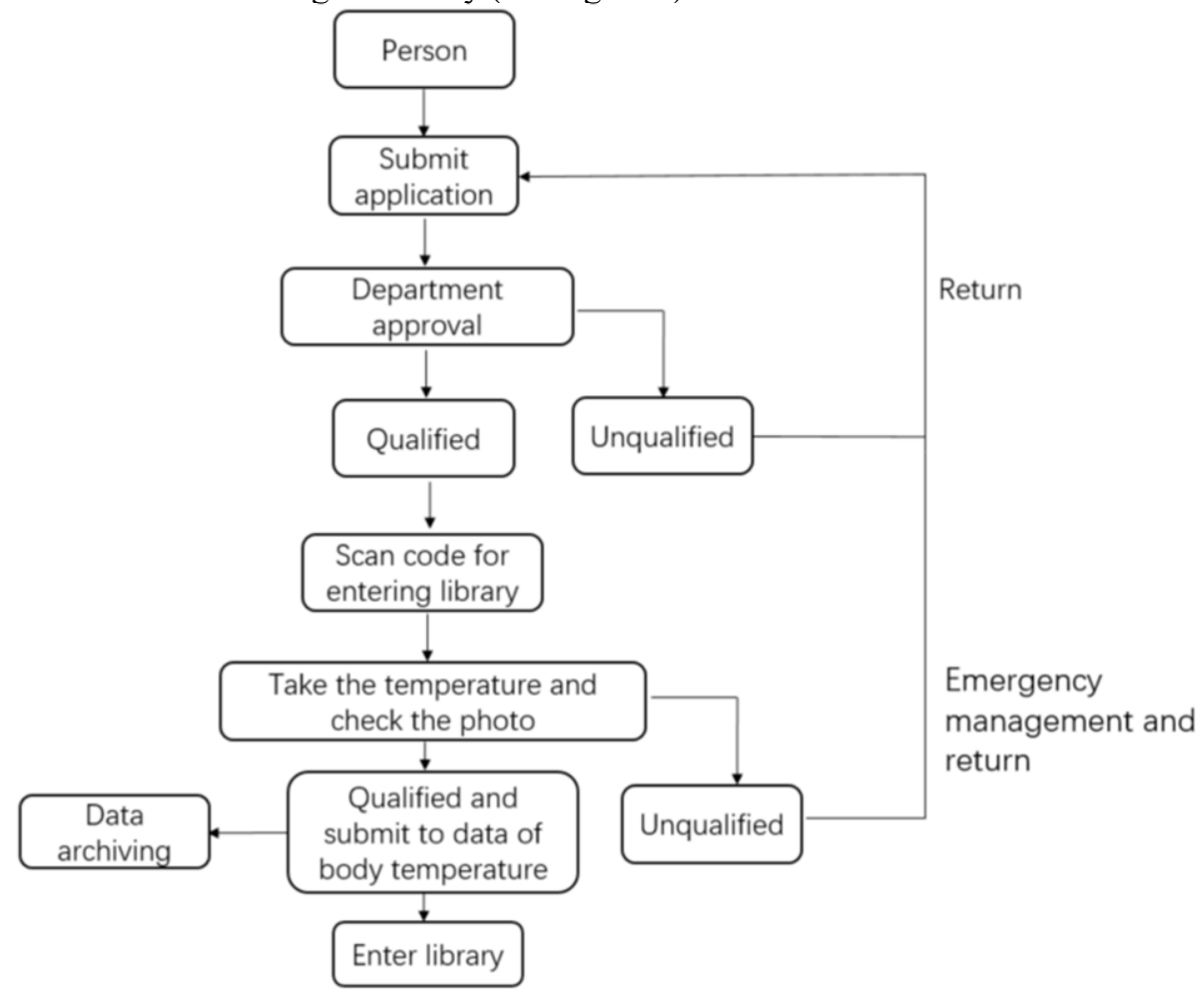

Figure 1. Flow chart of admission approval.

\subsubsection{System functions design.}

According to the needs of the epidemic situation, the system mainly includes five functional modules:

- user login,

- application management,

- QR code management,

- entering library record management,

- and system management.

Each module carries out strict operation control according to different usepermission of users. And sub modules for each module have been designed and developed as the following:

- the user login module includes two sub modules: system login and WeChat login;

- the application management module includes four sub modules: new application, revise application, approval and refuse;

- QR code management module includes two sub modules: new code and setting code; 
- entering library record management module includes four sub modules: scan code, record body temperature, record query and record export;

- and the system management module includes two sub modules: user management and department management.

The system function design block diagram can be seem from figure 2 .

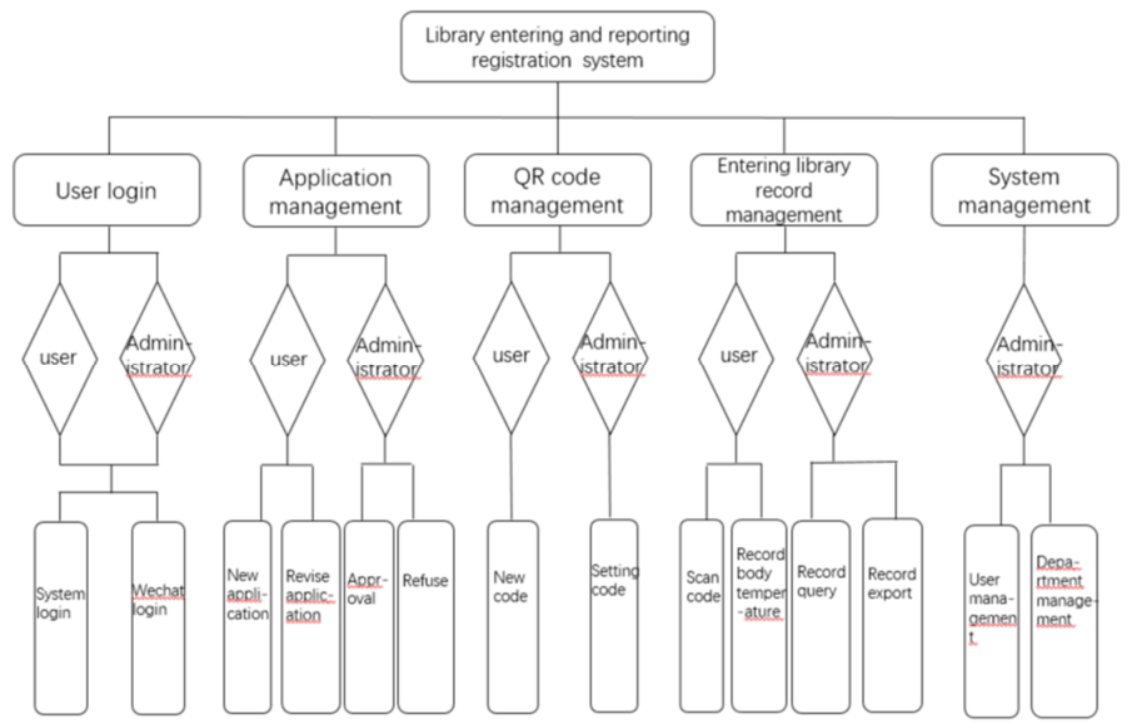

Figure 2. System function design block diagram.

\section{$4 \quad$ Results}

In this part, four functional sub modules which include WeChat login in the user login module, new code in the QR code management module, scan code in the entering library record management module, and batch export of record data to Excel file have been taken as examples to illustrate the implementation of this system.

\subsection{Database table design}

The design of database table structure is an important step in database development. Whether the model structure design is reasonable directly affects the efficiency of subsequent program development. According to the business process and specific requirements of the system, database tables such as user information table, application information table, QR code information table and entering library information table should be established in the database. Taking "user information table" as an example, the "user information table" is used to record the basic information of all users entering the library (see Table 1).

Table 1. User Information Table

\begin{tabular}{ccccc}
\hline Field name & Field type & Null & Constraint & Explanation \\
\hline uid & varchar(50) & no & primary key & staff id \\
uname & varchar(50) & no & $/$ & name \\
password & varchar(50) & no & $/$ & password \\
cellphone & varchar(50) & no & $/$ & mobile phone \\
\hline
\end{tabular}




\begin{tabular}{|c|c|c|c|c|}
\hline utype & int & no & foreign key & user type \\
\hline typename & $\operatorname{varchar}(50)$ & no & / & type name \\
\hline labid & int & no & foreign key & team id \\
\hline labname & $\operatorname{varchar}(50)$ & no & / & team name \\
\hline depid & int & no & foreign key & department id \\
\hline depname & $\operatorname{varchar}(50)$ & no & / & department name \\
\hline application & int & no & / & application or not \\
\hline lab_lead & int & no & I & leader or not \\
\hline lab_right & int & no & / & $\begin{array}{c}\text { department approval } \\
\text { auth }\end{array}$ \\
\hline dep_lead & int & yes & / & department leader \\
\hline dep_right & int & no & 1 & $\begin{array}{c}\text { department approval } \\
\text { auth }\end{array}$ \\
\hline admin & int & no & / & administrator \\
\hline photo & int & yes & / & photo image \\
\hline status & int & no & / & status \\
\hline
\end{tabular}

\subsection{Website form design of "application form for entering the library"}

For users first time entering the library, they must fill in and submit the application forms. The contents of the application form include: the location in recent 14 days, the physical condition of the recent 14 days, whether he/she has been to Hubei area in recent 14 days, whether he/she has been exposed to the person in Hubei area, and whether he/she has contacted the person of the Covid-19 and so on (see Figure 3).

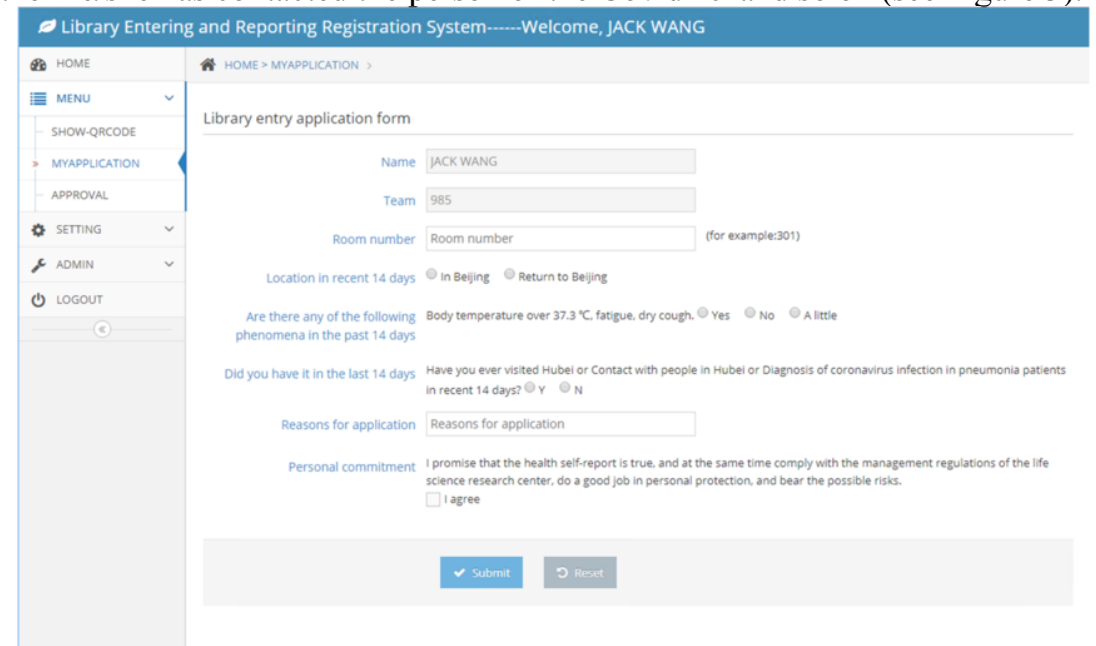

Figure 3. Application form for entering the library.

\subsection{Realization of system functions}

\subsubsection{Realization of login function based on WeChat authorization system.}

The key to login through WeChat is to get the OpenID of users. OpenID is the only sign of users under WeChat official account. With the help of the WeChat official account of the library, OpenID can be verified, and the authorized login system of WeChat can be realized. The specific steps include: (1) Generation of login QR code. According to the authorization interface provided by WeChat, the following URL is generated: 
https://open.weixin.qq.com/connect/oauth2/authorize?appid=wx4c4af2cf65b36bc2\&re direct_uri $=$ http $\% 3 \mathrm{~A} \% 2 \mathrm{~F} \% 2 \mathrm{Flibwx} . \mathrm{cau}$. edu.cn $\% 2 \mathrm{Fgetopenid.php \& response} \mathrm{type}=$ cod e\&scope $=$ snsapi_base\&state $=123 \& \#$ wechat_redirect. And convert this URL to QR code as login QR code. (2) User scanning code to login. Users can login to the system normally after passing the validity verification by scanning the WeChat code.

The realization of the function of generating $\mathrm{QR}$ code for entering the Library. After users login to the system by mobile phone, the system will automatically adapt to the resolution of the mobile phone, and automatically shrink the system menu bar to a pull-down menu bar. After clicking the button of "scan code to enter the library", the system will pop up the "enter the library QR code" window. This QR code is the only anti-counterfeiting QR code generated by RSA algorithm.

\subsubsection{Realization of the security client's function of scanning code and submitting body temperature.}

After security personnel scanning the anti-counterfeiting QR code, measuring the body temperature, checking the photos, and submitting the temperature information, the user may enter the library.

\subsubsection{Realization of batch export Excel file function of entering library record data.}

The batch export of record data in this system adopts LayUI Excel extension component, which generates Excel file and downloads it asynchronously through frontend technology AJAX, and the back-end program is only responsible for outputting corresponding JSON format data.

\section{Conclusion}

The development of the entering and reporting registration system platform of the CAU library has made full use of a large number of open-source frameworks and technologies after literature review, realized the functions of supporting the online use of a variety of client devices in the way of traditional web applications combined with mobile terminals, and ensured the timely and accurate information tracking management of the users entering the library in a special epidemic period. The system has the characteristics of high security, strong practicality and high scalability.

Since the system has put into operation, it has recorded and summarized all kinds of user information in detail during the epidemic period. It has achieved $100 \%$ of the library entrance registration. By the middle of June 2020, more than 2,300 user records have been leading into the system, with more than 2,700 users recorded for entering the library in total. On average, nearly 100 users enter the library each day.

The use of the system reduces the probability of personal contact, simplifies the process of manual management, improves work efficiency, and provides convenient conditions for follow-up information tracking, statistics and analysis. In addition to the CAU library, the system is also accepted by other departments and colleges at the University with excellent feedback from all of them.

With the increasing number of users registering to the system platform, the system functions still need to be optimized. For example, some functional modules of the system should be improved and modified according to the needs from different departments/colleges, adding the functions of request for withdrawal and verify refuse, 
the user-defined setting of $\mathrm{QR}$ code aging, the further refinement of user rights management, and the further strengthening of art design etc.

The development and utilization of the system platform for the CAU library will meet the actual needs of epidemic prevention and control in current special epidemic period, and play an extremely important role in ensuring the smooth progress of the scientific research, teaching and personal safety of staffs and students at the university.

\section{References}

[1] WHO, WHO Coronavirus Disease (COVID-19) Dashboard. WHO (2020), DOI=https://who.sprinklr.com/.

[2] D. Mehta and X. Wang, COVID-19 and digital library services - a case study of a university's library, Digital Library Perspectives, 2020, DOI=https://doi.org/10.1108/DLP-05-2020-0030

[3] L. Zach, What Do I Do in an Emergency? The Role of Public Libraries in Providing Information During Times of Crisis, Science \& Technology Libraries, 30:4 (2011), 404-413, DOI: 10.1080/0194262X.2011.626341.

[4] Y. H. Yin, et al. The System Architecture for the Face Recognition Based on Mixed Mode. Applied Mechanics and Materials, vol. 380-384, Trans Tech Publications, Ltd., Aug. (2013), 3791-3794. Crossref, DOI:10.4028/www.scientific.net/amm.380-384.3791.

[5] Z. J. Lu, Design and implementation of University Asset repair system based on B/S mode, Computer knowledge and technology 15 (2019) (36), 104-106.

[6] Y. Li, Building websites based on Linux, Apache, MySQL and PHP platforms, Electronic technology and software engineering 4 (2015),20.

[7] H. Shu, Y. F. Xiong and X. J. GE, Responsive web page design and Implementation Based on bootstrap framework, Journal of Beijing Printing Institute 24 (2016),47-52.

[8] P. Zhou, N. Zhao and M. Li, Application of bootstrap framework in responsive web design, Software Guide 16 (2017), 135-137.

[9] C. Cao and Z. G. Liu, Engineering science frontier and practice system based on SSH and layUI, Industrial control computer 32 (2019), 91-92.

[10] Q. S. Zheng \& X. M. Bi, Ajax Technology's Application and Research in Network Teaching Resources Library System. Applied Mechanics and Materials, 44-47 (2010), 3279-3283. DOI=https://doi.org/10.4028/www.scientific.net/amm.44-47.3279

[11] S. Peng, Data interactive application of report system based on jQuery framework, Non ferrous metal processing 47 (2018), 55-56.

[12] B. L. Xu, J. Luo and G. Pan, Application Research of data exchange technology based on, JSON Software Guide 16 (2017), 173-175.

[13] N. Q. Qi, Application research on RSA algorithm in QR code anti-counterfeiting technology, Nanjing University of Posts and Telecommunications (2017).

[14] W. Rahaman, Enhancing library services using Barcode, QR Code and RFID technology: a case study in Central Library National Institute of Technology, Rourkela, International Journal of Digital Library Services, vol. 6 (2016), 39-50.

[15] G. Z. Yi, Research and implementation of RSA algorithm, Modern computer (Professional Edition) 30 (2018), 12-14.

[16] J. Wang, LayUI-excel simple and quick export plug-in components. (2015) DOI=http://excel.wj2015.com/.

[17] X. W. Xu, Module and process design and implementation of science and technology novelty search system based on WeChat login, Tianjin Science and technology 46 (2019), 68-70. 